\title{
Rumenocentesis dorsomedial: un procedimiento seguro para la obtención de líquido ruminal en vacas lecheras a pastoreo
}

\author{
Dorsomedial rumenocentesis: a safe procedure for collecting ruminal fluid samples \\ from grazing dairy cows
}

\author{
M Noro ${ }^{\mathrm{a}}$, P Sepúlveda ${ }^{\mathrm{b}}$, F Cárdenas ${ }^{\mathrm{a}}$, RH Chihuailafa ${ }^{\mathrm{a}}$, F Wittwer ${ }^{\mathrm{a}}$ \\ anstituto de Ciencias Clínicas Veterinarias, Facultad de Ciencias Veterinarias, Universidad Austral de Chile, Valdivia, Chile. \\ bProg. Doc. Cs. Vet. Escuela de Graduados, Facultad de Ciencias Veterinarias, Universidad Austral de Chile, Valdivia, Chile.
}

\begin{abstract}
SUMMARY
Two experiments were carried out to 1) compare $\mathrm{pH}$ values of rumen fluid samples (RF) obtained from dairy cows by dorsomedial rumenocentesis, a probe and permanent cannula and 2) to evaluate the effectiveness of the rumenocentesis and its effects on the health and performance of HolsteinFrisian grazing dairy cows. Experiment 1: Four lactating grazing Holstein-Frisian cows with a permanent rumen cannula were used. RF samples were obtained at 8:30, 13:30 and 17:30 hours, using a probe and the cannula (from caudoventral, caudodorsal, ventral and dorsal sacs). A RF sample was also obtained at 17:30 hours by dorsomedial rumenocentesis. $\mathrm{pH}$ of the samples obtained by rumenocentesis and from the caudoventral sac were similar and correlated $(\mathrm{r}=0.77)$. Experiment 2: A dorsomedial rumenocentesis was performed in 30 grazing lactating Holstein-Frisian cows. The procedure was repeated every 5 days during one month and its effectiveness and effect on the health and performance of the cows were evaluated. The rumenocentesis was effective to obtain $\mathrm{RF}$ samples for $\mathrm{pH}$ determination $(>3 \mathrm{~mL})$ in all cases $(\mathrm{n}=150)$. Reactions movements to the procedure were within accepted limits and more intensive at the first attempts. The performance and rectal temperature of the cows were not affected. A temporary swelling (1.0-1.5 cm) on the area was appreciated in $4 \%$ of the cases. It is concluded that dorsomedial rumenocentesis is a practical and safe procedure to obtain RF samples in grazing dairy cows under field conditions to evaluate the acid base balance of the rumen.
\end{abstract}

Palabras clave: vacas, líquido ruminal, rumenocentesis, acidosis ruminal subaguda.

Key words: cows, rumen fluid, rumenocentesis, subacute rumen acidosis.

\section{INTRODUCCIÓN}

La intensificación de las prácticas ganaderas en sistemas pastoriles de países con clima templado ha favorecido la presentación de trastornos metabólico-nutricionales en los rebaños lecheros, entre los que destacan desequilibrios como la acidificación del pH ruminal (Westwood y col 2003, Noro y col 2010). Esta situación es condicionada por la ingesta de forrajes tiernos, de alta calidad, con contenidos altos de agua y bajos en fibra efectiva, situación que favorece una rápida degradación ruminal con producción de ácidos en el rumen (Westwood y col 2003).

El pH ruminal es dependiente de la tasa de producción y absorción de ácidos producidos en el rumen, mayormente los ácidos grasos volátiles (AGV) acético, propiónico y butírico, complementado por la acción de tampones o amortiguadores de $\mathrm{pH}$, mayormente aportados por la saliva (Dohme y col 2008). Es así que el pH ruminal varía entre 0,5 a 1,0 unidad durante el transcurso del día, siendo el punto de mayor acidez después de 2 a 4 horas posingesta

Aceptado: 25.10.2012.

* Hospital Veterinario, Campus Isla Teja s/n, Valdivia, Chile; mirelanoro @ gmail.com de alimentos ricos en carbohidratos altamente fermentables (Oetzel 2003). El pH del líquido ruminal (LR) de las vacas en pastoreo disminuye a medida que progresan las horas del día (Scandolo y col 2007), probablemente asociado a la periodicidad de la ingesta de forrajes y a los periodos de rumia (Noro y col 2011). El valor de pH es más bajo entre las 16:00 y las 18:00 horas, cuando la ingesta de materia seca de alta digestibilidad es mayor (Wales y col 2004), lo que provoca una mayor fermentación ruminal, producción de $\mathrm{CO}_{2}$ y de AGV (Kolver y de Veth 2002).

Para que la vaca conserve una alta productividad se requiere que el $\mathrm{pH}$ del LR se mantenga dentro de valores que permitan una adecuada digestión de los forrajes, siendo óptimo un $\mathrm{pH}$ de 6,35. No obstante, producto del efecto negativo de la digestión de la celulosa asociada al menor desarrollo de las bacterias celulolíticas, la digestibilidad de la fibra disminuye con valores de $\mathrm{pH}$ inferiores a 5,8 (Kolver y de Veth 2002, Wales y col 2004). Por otro lado, la presentación de acidosis subaguda (SARA, $\mathrm{pH}$ ruminal $\leq 5,5$ ) en vacas lecheras en condiciones de pastoreo se ha descrito en Irlanda (O'Grady y col 2008), Nueva Zelandia (Westwood y col 2003) y Chile (Noro y col 2010), la cual puede afectar la fermentación ruminal, generar pérdidas en el bienestar animal y la productividad y rentabilidad pecuaria (Morgante y col 2007). 
La determinación del pH del LR es el único indicador directo del equilibrio ácido-básico ruminal en un rebaño, siendo necesario para ello obtener muestras a campo. A su vez, el pH ruminal varía en los diferentes lugares dentro del rumen (Garrett y col 1999), por lo que la evaluación del pH del líquido ruminal debería efectuarse desde la misma región del rumen en cada muestreo. En condiciones de campo, las muestras de LR pueden ser obtenidas mediante la sonda ororruminal y por rumenocentesis. En sistemas con manejo intensivo con alimentación basada en granos la obtención de muestras de LR por medio de rumenocentesis tiene una especificidad similar a la sonda ororruminal, pero una sensibilidad mayor (Duffield y col 2004). Sin embargo, la muestra obtenida con sonda contamina con saliva alterando los valores de $\mathrm{pH}$ (Garrett y col 1999), por lo que se recomienda el uso de rumenocentesis como prueba de campo para el diagnóstico de SARA (Duffield y col 2004). En la rutina, la técnica de rumenocentesis ventral es la más utilizada, pero en los sistemas pastoriles presenta dificultades de ejecución debido al diseño de las instalaciones y al temperamento de los animales, por lo que se considera conveniente validar una técnica de rumenocentesis dorsomedial que pueda ser realizada de forma efectiva y segura en vacas contenidas en una manga.

Por otro lado, algunos autores indican que el $\mathrm{pH}$ fecal (Clayton y col 1999) y urinario (Bouda y col 1997) se correlacionan con el $\mathrm{pH}$ ruminal, motivo por el cual podrían constituir una herramienta para el diagnóstico de acidosis ruminal.

En consideración a estos antecedentes, los objetivos de este estudio fueron comparar los valores de $\mathrm{pH}$ del LR obtenido por sonda ororruminal y rumenocentesis dorsomedial con muestras obtenidas directamente del rumen en vacas fistuladas y correlacionar los valores de $\mathrm{pH}$ ruminal con los valores de $\mathrm{pH}$ fecal y urinario, así como evaluar el éxito y seguridad de esta técnica realizada repetidamente a vacas lecheras en lactancia.

\section{MATERIAL Y MÉTODOS}

Se realizaron dos experimentos durante la primavera en el rebaño lechero de la estación experimental de la Universidad Austral de Chile, Valdivia, $3^{\circ} 46^{\prime}$ LS y $73^{\circ} 13^{\prime}$ LO, Región de Los Ríos, Chile.

\section{EXPERIMENTO 1}

Animales y alimentación. Se emplearon cuatro vacas Holstein-Frisian fistuladas con cánulas ruminales permanentes, con un peso medio de $500 \pm 50 \mathrm{~kg}$, de 3 a 5 años de edad, entre el $3^{\circ}$ y $5^{\circ}$ mes de lactancia y con una producción de leche entre 25 y $30 \mathrm{~L} /$ día. Las vacas fueron mantenidas en pastoreo rotativo de praderas permanentes con predominio de Lolium perenne y Bromus valdivianus, y libre acceso a agua en potreros de 1 a 4 ha de superficie y con sistema de rotación en franjas asignadas dos veces al día. Además, cada animal recibió durante la ordeña de la mañana (8:00 horas [h]) y tarde $(17: 00 \mathrm{~h}) 2,5 \mathrm{~kg}$ de concentrado comercial (Suralim Mega 2132, IANSAGRO) para vaca en lactancia.

Obtención y análisis de muestras. Se realizaron cinco muestreos con intervalos de tres días, en los que se obtuvieron muestras de LR en tres oportunidades: 8:30, 13:30 y 17:30 h. En cada oportunidad se obtuvo una muestra de LR mediante sonda ororruminal y cuatro de distintas zonas del rumen (craneoventral, craneodorsal, caudoventral y caudodorsal) manualmente a través de la cánula ruminal. Además, se obtuvo una muestra de LR mediante una rumenocentesis dorsomedial realizada a las 17:30 h, siendo esta lograda previo a las obtenidas mediante cánula para mantener la anaerobiosis ruminal. También se recolectaron muestras de heces y orina en los mismos tiempos y días de los muestreos de LR.

La obtención de LR con sonda ororruminal se realizó con una sonda de $2,0 \mathrm{~m}$ de largo y $1,5 \mathrm{~cm}$ de diámetro, la que fue introducida vía oral protegida a través de un abreboca. La aspiración del LR se realizó por succión.

La rumenocentesis dorsomedial se realizó puncionando con una aguja $14 \mathrm{G}$ de $10 \mathrm{~cm}$ de largo en la fosa paralumbar izquierda, 15 a $20 \mathrm{~cm}$ ventral a los procesos transversos de las vértebras lumbares previa tricotomía y desinfección de la zona con alcohol yodado. La aguja se dirigió en sentido ventral, para ubicarla en un punto entre los sacos caudoventral y caudodorsal (figura 1); seguidamente se conectó a la aguja una jeringa de $10 \mathrm{~mL}$ y se aspiró hasta obtener un volumen de 3 a $6 \mathrm{~mL}$ de LR.

Inmediatamente de obtenida la muestra se retiró la aguja y el émbolo del cuerpo de la jeringa y se sumergió el electrodo del $\mathrm{pH}$-metro en la muestra para obtener la lectura del pH (pH-metro digital Checker 3.0 Hanna

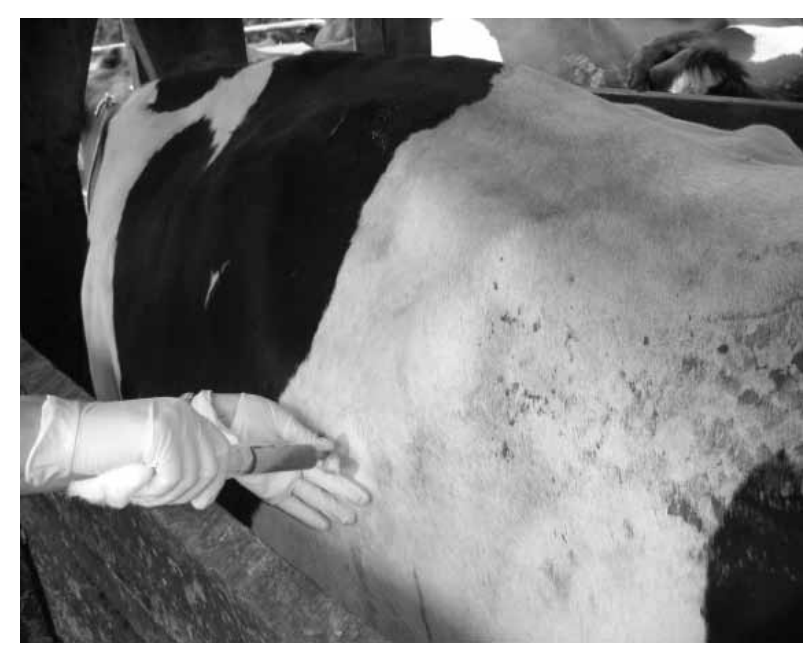

Figura 1. Zona de punción durante la ejecución de la rumenocentesis dorsomedial en una vaca contenida en manga.

Puncture area of the dorsomedial rumenocentesis technique for a cow in a chute. 
Instruments $\left.{ }^{\circledR}\right)$. Además, se determinó el tiempo de reducción de azul de metileno (TRAM) en las muestras de LR obtenidas a la 17:30 h mediante sonda ororruminal, cánula (desde el saco caudoventral) y rumenocentesis, incubando a $37^{\circ} \mathrm{C}$ una alícuota de $2,5 \mathrm{~mL}$ de cada muestra en un tubo de ensayo y agregando $0,125 \mathrm{~mL}$ de azul de metileno al 0,03\% (Wittwer 2009).

Análisis estadístico. Los datos fueron evaluados en el programa Statistix 8.0 (Analytical Software, Roseville, MN, USA) en cuanto a normalidad por la prueba de Shapiro-Wilk y homocedasticidad mediante la prueba de Barttlet. Las variables homocedásticas fueron comparadas mediante ANDEVA $\left(\mathrm{Y}_{i}=\mu+\mathrm{M}_{i}+\varepsilon_{i}\right.$; donde $\mu=$ media general, $\mathrm{Mi}=$ método de obtención de la muestra, $\varepsilon \mathrm{i}=$ error experimental) y las heterocedásticas mediante KruskallWallis. Se correlacionaron los valores de $\mathrm{pH}$ ruminal entre las muestras obtenidas por sonda ororruminal mediante cánula y rumenocentesis a través de la prueba de Spearman. Se consideraron significativas las diferencias cuando el valor de $\mathrm{P}$ fue inferior a 0,05 .

\section{EXPERIMENTO 2}

Animales y alimentación. Se emplearon 30 vacas HolsteinFrisian con un peso vivo de $532 \pm 41 \mathrm{~kg}$, de 3 a 5 años de edad, entre el $3^{\circ}$ y $5^{\circ}$ mes de lactancia y con una producción de leche entre 25 y 30 L/día. La alimentación fue similar a las vacas del experimento 1, salvo que los horarios de ordeña y entrega de concentrado fueron a las 6:30 y 15:30 h.

Obtención y análisis de muestras. A cada vaca se le realizaron 5 rumenocentesis dorsomedial posterior a la ordeña de la tarde (17:00 h), con intervalos de cinco días entre ellas. Al mismo tiempo se obtuvieron muestras de materia fecal y orina mediante recolección rectal y estimulación subvulvar, respectivamente. Inmediatamente posterior a la obtención de cada muestra se determinó el valor de $\mathrm{pH}$ mediante el uso de un $\mathrm{pH}$-metro digital portátil Checker 3.0 Hanna Instruments ${ }^{\circledR}$.

Evaluación de la rumenocentesis. Se estableció el éxito de la rumenocentesis al obtener un mínimo de $3 \mathrm{~mL}$ de LR y se evaluó el comportamiento de las vacas durante su ejecución utilizando la escala corta de Glasgow modificada (Murrell y col 2008) (cuadro 1).

Evaluación del estado de salud. La temperatura rectal de cada vaca se estableció 24 h previo a la realización de la rumenocentesis y a las 24 y $48 \mathrm{~h}$ posteriores a ésta. La producción diaria de leche se determinó tres veces a la semana durante todo el período del ensayo hasta las $48 \mathrm{~h}$ posterior a la última rumenocentesis mediante un medidor Waikato $42 \mathrm{~kg}$. Además, se evaluó la presencia de aumentos de volumen y la respuesta de los animales a la presión ejercida sobre la región de la rumenocentesis (cuadro 2).
Cuadro 1. Escala corta de Glasgow modificada (Murrell y col 2008).

Short form of the Glasgow scale (Murrell y col 2008).

\begin{tabular}{lc}
\hline $\begin{array}{l}\text { Comportamiento de la vaca } \\
\text { durante la rumenocentesis: }\end{array}$ & Puntaje \\
\hline Indiferente & 0 \\
Movimiento calmo & 1 \\
Movimiento moderado & 2 \\
Movimientos bruscos & 3 \\
Patea & 4 \\
\hline
\end{tabular}

Cuadro 2. Aumentos de volumen y respuesta de los animales a la presión ejercida sobre la región de la rumenocentesis. Volume increase and response of the animals to pressure on the rumenocentesis area.

\begin{tabular}{lr}
\hline Al ejercer una presión moderada a $5 \mathrm{~cm}$ del sitio de punción: \\
\hline No hace nada & 0 \\
Vuelve la vista & 1 \\
Intenta retirarse & 2 \\
Patea & 3 \\
Vocaliza & 4 \\
\hline
\end{tabular}

Análisis estadístico. Los datos fueron evaluados en el programa Statistix 8.0 (Analytical Software, Roseville, MN, USA). Se asociaron los valores de $\mathrm{pH}$ ruminal, urinario y fecal mediante correlación de Spearman. La respuesta de los animales a la rumenocentesis entre los días de muestreo fue comparada mediante la prueba de Chi-cuadrado. Se consideraron significativas las diferencias cuando el valor de $\mathrm{P}$ fue inferior a 0,05 .

\section{RESULTADOS}

\section{EXPERIMENTO 1}

Los valores de $\mathrm{pH}$ de las muestras de LR obtenidas a las 8:30 h del saco craneodorsal presentaron valores superiores a las de los sacos craneoventral, caudodorsal y caudoentral ( $\mathrm{P}<0,05$; figura 2 ). A su vez, los valores de $\mathrm{pH}$ de las muestras obtenidas por sonda ororruminal así como de los diferentes sacos del rumen fueron similares en los muestreos de las 8:30, 13:30 y 17:30 h ( $\mathrm{P}>0,05$, figura 2).

Los valores de $\mathrm{pH}$ de las muestras obtenidas mediante sonda ororruminal fueron superiores $(\mathrm{P}<0,05$, figura 2$)$ en aproximadamente 1,0 punto a los obtenidos a través de la cánula y por rumenocentesis, con valores máximos de $\mathrm{pH}=9,0$. A su vez, los valores de $\mathrm{pH}$ ruminal de las muestras obtenidas mediante sonda ororruminal presentaron una alta correlación con el de las muestras obtenidas del 
saco craneoventral $(r=0,72)$ y baja correlación respecto de las muestras obtenidas del saco craneodorsal, caudodorsal y caudoventral (cuadro 3 ).

Los valores de $\mathrm{pH}$ del LR de las muestras obtenidas mediante rumenocentesis dorsomedial presentaron valores similares a los de los distintos sacos del rumen $(\mathrm{P}>0,05$, figura 2) y se correlacionaron mejor con los valores obtenidos del saco caudoventral del rumen (figura 3a), seguida por los del saco craneoventral (figura $3 \mathrm{c}$ ), saco craneodorsal (figura 3d) y del saco caudodorsal (figura 3b), y con baja correlación con los valores de muestras obtenidas por sonda ororruminal (cuadro 1; figura 2e).

El TRAM fue más lento en las muestras obtenidas mediante sonda ororruminal $(\mathrm{P}<0,05)$ que en las muestras obtenidas del saco caudoventral del rumen y mediante rumenocentesis, las cuales fueron similares entre sí $(\mathrm{P}>0,05)$, incluso en algunas muestras obtenidas por sonda ororruminal $(n=25)$ no se produjo la reducción del azul de metileno (figura 4).

\section{EXPERIMENTO 2}

La rumenocentesis dorsomedial permitió obtener volúmenes de líquido ruminal superiores a $3 \mathrm{~mL}$ en todas las punciones. Durante el procedimiento la mayoría de las

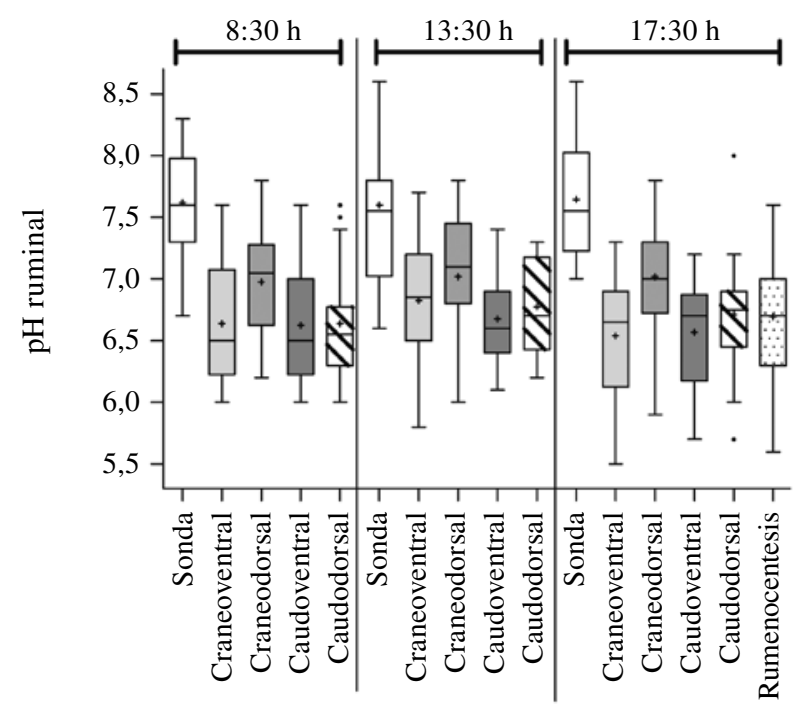

Figura 2. Gráfico de caja y bigotes de los valores de $\mathrm{pH}$ de líquido ruminal obtenidos a las 8:30, 13:30 y 17:30 h mediante sonda (sonda ororruminal), cánula ruminal (desde los sacos craneoventral, craneodorsal, caudoventral y caudodorsal del rumen) y rumenocentesis dorsomedial (solo a las 17:30 h). $+=$ media.

Box plot for $\mathrm{pH}$ values of ruminal fluid samples obtained at $8: 30,13: 30$ and $17: 30 \mathrm{~h}$ by a probe, permanent cannula (caudoventral, caudodorsal, ventral and dorsal ruminal sacs) and dorsomedial rumenocentesis (only 17:30 h). $+=$ mean .

Cuadro 3. Valores de correlación de Spearman y significancias (valor de P) entre los valores de pH de líquido ruminal obtenidos mediante sonda ororruminal, cánula ruminal (sacos craneoventral, craneodorsal, caudoventral y caudodorsal) y rumenocentesis dorsomedial.

Spearman correlation values and significance ( $\mathrm{P}$ value) between $\mathrm{pH}$ values of ruminal fluid samples obtained by a probe, permanent cannula (caudoventral, caudodorsal, ventral and dorsal ruminal sacs) and dorsomedial rumenocentesis).

\begin{tabular}{|c|c|c|c|c|c|}
\hline & Sonda & Craneodorsal & Craneoventral & Caudodorsal & Caudoventral \\
\hline Craneodorsal & $\begin{array}{c}0,5590 \\
(0,0000)\end{array}$ & & & & \\
\hline Craneoventral & $\begin{array}{c}0,7231 \\
(0,0000)\end{array}$ & $\begin{array}{c}0,7555 \\
(0,0000)\end{array}$ & & & \\
\hline Caudodorsal & $\begin{array}{c}0,4546 \\
(0,0012)\end{array}$ & $\begin{array}{c}0,7490 \\
(0,0000)\end{array}$ & $\begin{array}{c}0,7460 \\
(0,0000)\end{array}$ & & \\
\hline Caudoventral & $\begin{array}{c}0,5849 \\
(0,0000)\end{array}$ & $\begin{array}{c}0,6810 \\
(0,0000)\end{array}$ & $\begin{array}{c}0,7785 \\
(0,0000)\end{array}$ & $\begin{array}{c}0,7248 \\
(0,0000)\end{array}$ & \\
\hline Rumenocentesis & $\begin{array}{c}0,4443 \\
(0,0971)\end{array}$ & $\begin{array}{c}0,6632 \\
(0,0070)\end{array}$ & $\begin{array}{c}0,7613 \\
(0,0002)\end{array}$ & $\begin{array}{c}0,5929 \\
(0,0198)\end{array}$ & $\begin{array}{c}0,7734 \\
(0,0001)\end{array}$ \\
\hline
\end{tabular}

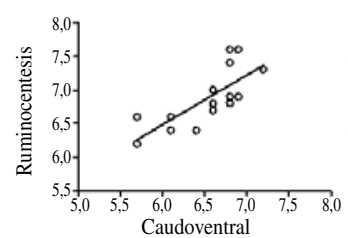

a

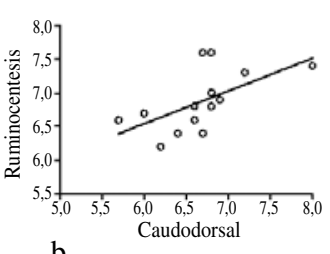

$\mathrm{b}$

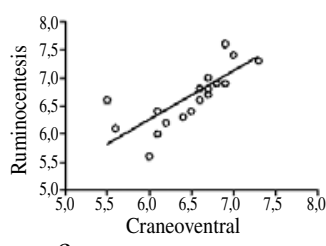

$\mathrm{c}$

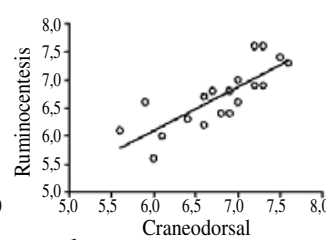

d

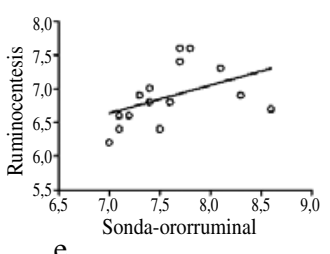

$\mathrm{e}$

Figura 3. Líneas de regresión entre $\mathrm{pH}$ del líquido ruminal de muestras obtenidas mediante rumenocentesis y cánula ruminal desde los sacos caudoventral (a), caudodorsal (b), craneoventral (c) y craneodorsal (d) y mediante sonda ororruminal (e).

Regressions lines between the $\mathrm{pH}$ values of ruminal fluid samples obtained by dorsomedial rumenocentesis, permanent cannula (caudoventral (a), caudodorsal (b), ventral (c), dorsal ruminal sacs (d) and a probe (e). 
vacas realizó movimientos en la manga o pateó, respuestas que fueron más bruscas durante la primera rumenocentesis (día 0), en la cual ningún animal permaneció indiferente a la punción (cuadro 2).

Ningún animal tuvo baja en la producción láctea o alza térmica los días posteriores a la rumenocentesis que indique cambios patológicos. De las 150 rumenocentesis, solo en seis vacas se observaron aumentos temporales de volumen $(\sim 1-1,5 \mathrm{~cm})$ en el sitio de la punción, tres posterior a la $2^{\mathrm{a}}$ punción y tres posterior a la $3^{\mathrm{a}}$ punción, de las cuales dos de la $2^{\mathrm{a}}$ y dos de la $3^{\mathrm{a}}$ punción presentaron contenido purulento. Todas las lesiones desaparecieron antes del término del estudio.

Los valores de $\mathrm{pH}$ ruminal de las muestras obtenidas mediante rumenocentesis presentaron un baja correlación con el pH fecal (figura 5a) y no se correlacionaron con los de $\mathrm{pH}$ urinario (figura 5b). Tampoco se observó correlación entre el $\mathrm{pH}$ fecal y urinario (figura $5 \mathrm{c}$ ).

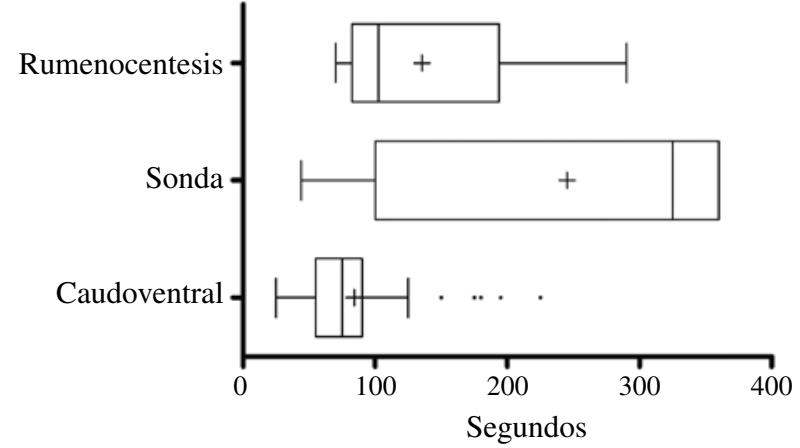

Figura 4. Gráfico de caja y bigotes del tiempo de reducción de azul de metileno en muestras de líquido ruminal obtenidas mediante rumenocentesis, sonda y cánula ruminal (saco caudoventral) de vacas lecheras a pastoreo.

Box plot for the methylene blue reduction time of ruminal fluid samples obtained from grazing dairy cows by dorsomedial rumenocentesis, probe, and permanent cannula (caudoventral ruminal sac).
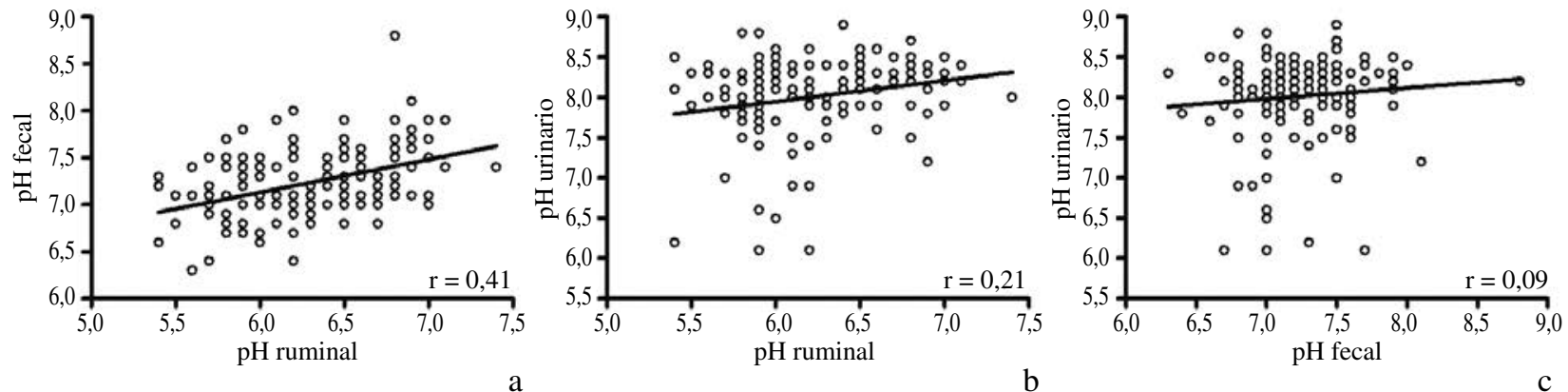

Figura 5. Curva de regresión entre $\mathrm{pH}$ del líquido ruminal de vacas lecheras a pastoreo obtenido mediante rumenocentesis con los del $\mathrm{pH}$ fecal (a) y urinario (b) y entre $\mathrm{pH}$ fecal y urinario (c).

Regression lines between the $\mathrm{pH}$ values of ruminal fluid samples obtained by dorsomedial rumenocentesis and faeces and urine samples.

Los valores medios de $\mathrm{pH}$ de las muestras de orina fueron mayores en 0,8 puntos a las muestras de heces y 1,8 puntos mayores al de las muestras de LR obtenido mediante rumenocentesis (figura 6).

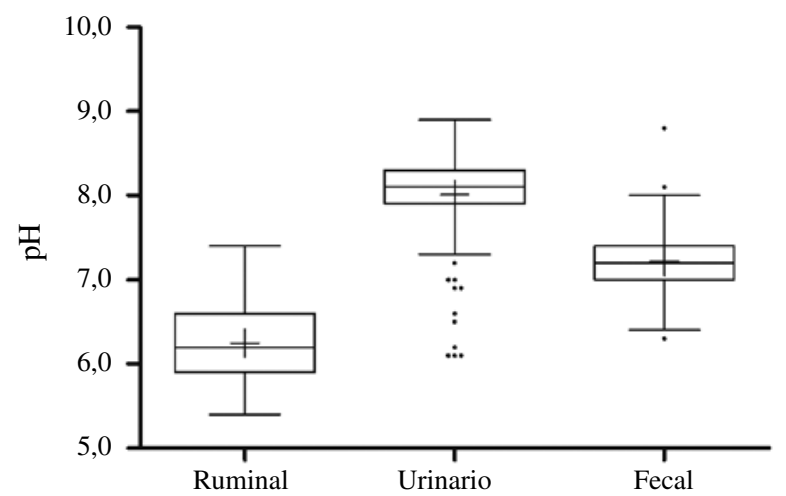

Figura 6. Gráfico de caja y bigotes de los valores de $\mathrm{pH}$ del líquido ruminal (rumenocentesis dorsomedial), orina y heces de vacas lecheras en pastoreo.

Box plot for $\mathrm{pH}$ values of ruminal fluid, urine and faeces samples from grazing dairy cows.

\section{DISCUSIÓN}

\section{EXPERIMENTO 1}

La diferencia en los valores de $\mathrm{pH}$ en las muestras obtenidas mediante sonda ororruminal de $+1,0$ punto es concordante con lo observado en otro estudio en el cual se observaron diferencias entre 0,6 a 1,7 puntos de $\mathrm{pH}$ (Nordlund y Garrett 1994), situación explicable por la contaminación con saliva de la muestra obtenida mediante sonda (Dirksen y Smith 1987). Además, en las muestras obtenidas con sonda no se tiene control del sitio de recolección en el rumen, con lo cual el valor de $\mathrm{pH}$ puede variar en cada recolección (Nordlund y Garrett 1994). Estos resultados permiten inferir que la muestra de LR obtenida mediante sonda no es adecuada para el diagnóstico de trastornos acidobásico ruminales.

La asociación entre los valores de $\mathrm{pH}$ del LR de las muestras obtenidas mediante rumenocentesis dorsomedial con el de los sacos caudoventral y caudodorsal del rumen concuerda con lo observado en muestras obtenidas mediante rumenocentesis ventral y directamente del saco 
caudoventral del rumen ( $r=0,72)$, (Garrett y col 1999). Estos resultados validan el uso de la rumenocentesis dorsomedial, si bien la muestra más representativa para la determinación de $\mathrm{pH}$ debería ser una muestra compuesta colectada desde varios sitios del rumen, o desde el centro del rumen, muestras que solo pueden ser colectadas en condiciones experimentales (Garrett y col 1999).

La rumenocentesis se realizó posterior al ordeño de la tarde basado en que el $\mathrm{pH}$ ruminal disminuye durante el transcurso del día de 6,7 a las 8:00 h a 5,9 a las 19:00 h en vacas a pastoreo y suplementadas con concentrado (Scandolo y col 2007). Los valores obtenidos en las muestras recolectadas en la mañana no difirieron mayormente con las de la tarde, lo que sugiere que eventualmente se podrían colectar muestras de LR en diferentes horarios del día. Sin embargo, se considera que el horario de la tarde, posterior al ordeño, es el más indicado para realizar la rumenocentesis dorsomedial como técnica diagnóstica de SARA.

Los valores de TRAM de las muestras obtenidas mediante sonda tuvieron valores superiores a los obtenidos mediante las otras técnicas, explicable por la contaminación con saliva en la colección con sonda, que altera el $\mathrm{pH}$ óptimo de reacción reductora de las bacterias ruminales (Dirksen y Smith 1987). La alta correlación observada entre los valores de $\mathrm{pH}$ de las muestras obtenidas mediante rumenocentesis con el obtenido directamente desde el rumen permite concluir que las muestras obtenidas mediante rumenocentesis son adecuadas para diagnosticar alteraciones acidobásicas ruminales en vacas lecheras.

\section{EXPERIMENTO 2}

En las 150 rumenocentesis realizadas se logró obtener una muestra de LR adecuada para determinar el $\mathrm{pH}$, demostrándose que esta técnica puede ser utilizada con dicho propósito en vacas. Se observó que las reacciones de respuesta a la punción disminuyeron posterior a punciones seriadas, aspecto no evaluado en estudios previos en los que se ha realizado rumenocentesis, probablemente porque en la mayoría de ellos se ha usado xilazina como sedante para facilitar el procedimiento (Nordlund y Garrett 1994, Garrett y col 1999). Las reacciones de rechazo fueron más bruscas en las primeras rumenocentesis $(\mathrm{P}<0,05)$, indicando que relacionaron más al contacto con personas que con la punción en sí.

El procedimiento de la rumenocentesis no afectó negativamente la producción de las vacas, así como no afectó su salud, antecedentes que avalan su inocuidad, similar a lo descrito en experiencias similares con esta técnica (Gianesella y col 2010). La aparición de 4\% de pequeños aumentos de volumen en el sitio de punción es superior al 1 a $2 \%$ reportado (Nordlund y Garrett 1994), lo que estaría asociado a la realización de rumenocentesis repetidas en el presente ensayo.
El pH urinario medio de 8,0 está en el límite superior del intervalo fisiológico (Bouda y col 1997), asociado a la ingesta de pradera con alto contenido de cationes (Anrique y col 2010). La baja correlación de los pH urinario y fecal con el del LR indica que para el diagnóstico de alteraciones acidobásicas ruminales no es adecuada la medición del pH urinario o fecal, aunque la observación de las características físicas de la materia fecal constituye una herramienta auxiliar en el diagnóstico de acidosis (Garry 2002). Hay que considerar que los valores de $\mathrm{pH}$ ruminal obtenidos en las vacas del estudio se mantuvieron dentro de los límites de referencia, situación que no descarta una eventual correlación entre los valores de pH del LR con los fecales (Maruta y Ortolani 2002) y urinarios (Maruta y col 2008), en animales con acidosis láctica aguda.

La técnica de rumenocentesis es dependiente del tipo y comportamiento de los animales, de su manejo y de las construcciones utilizadas en una zona. Bajo estas consideraciones, la técnica de rumenocentesis dorsomedial se ha visualizado como la más adecuada para usar en las vacas de rebaños a pastoreo mantenidas dentro de mangas de contención.

Se puede concluir que la rumenocentesis dorsomedial es una técnica práctica y segura para la obtención de una muestra representativa del líquido ruminal, pudiendo ser utilizada para la determinación del equilibrio acidobásico ruminal en vacas bajo condiciones de pastoreo, entregando valores de $\mathrm{pH}$ similares al LR del saco caudoventral del rumen. No se observó asociación entre pH ruminal, fecal y urinario bajo las condiciones del ensayo.

\section{RESUMEN}

Los objetivos del estudio fueron 1) comparar valores de $\mathrm{pH}$ de muestras de líquido ruminal (LR) obtenidas mediante rumenocentesis dorsomedial, sonda ororruminal, y directamente del rumen y 2) evaluar la efectividad de la rumenocentesis dorsomedial, mediante dos experimentos en vacas Holstein-Frisian en lactancia mantenidas a pastoreo. Experimento 1: en cuatro vacas fistuladas se obtuvieron muestras de LR a las 8:30, 13:30 y 17:30 horas mediante sonda ororruminal y por cánula ruminal (sacos caudoventral, caudodorsal, craneoventral y craneodorsal); además, de otra muestra obtenida a las 17:30 horas mediante rumenocentesis dorsomedial. Los $\mathrm{pH}$ del LR obtenidos mediante rumenocentesis y del saco caudoventral fueron similares y correlacionados $(r=0,77)$. Experimento 2: a 30 vacas Holstein-Frisian en lactancia y mantenidas en pastoreo se realizó una rumenocentesis dorsomedial cada cinco días, durante un mes, para evaluar la eficiencia del procedimiento y sus eventuales efectos en la salud y producción. En las 150 rumenocentesis ejecutadas se obtuvo una muestra de LR adecuada para determinar $\mathrm{su} \mathrm{pH}$ ( $>3 \mathrm{~mL}$ ), con manifestaciones de rechazo más bruscas en los primeros procedimientos. No se produjeron alzas térmicas o disminución de la producción láctea y en la zona de punción solo se apreció un aumento de volumen temporal de $1-1,5 \mathrm{~cm}$ en $4 \%$ de las vacas. Se concluye que la rumenocentesis dorsomedial es una técnica práctica y segura para la obtención de una muestra representativa del LR, pudiendo ser utilizada para la determinación del equilibrio ácido base ruminal en condiciones de pastoreo. 


\section{AGRADECIMIENTOS}

Al Proyecto FIA-CS-C-2004-1-P-002 M2P6, por financiar el estudio. Al Dr. Rubén Pulido por autorizar el uso de las vacas fistuladas y a Pilar Cerna por su apoyo en los muestreos.

\section{REFERENCIAS}

Anrique R, R Fuschlocher, S Iraira, R Saldaña. 2010. Composición de alimentos para el ganado bovino. Imprenta América, Valdivia, Chile.

Bouda J, M Paasch, O Yabuta. 1997. Desarrollo y empleo del método de diagnóstico preventivo de los trastornos ruminales y metabólicos en bovinos. Vet Mex 28, 189-195.

Clayton EH, IJ Lean, JB Rowe, JW Cox. 1999. Effects of feeding virginiamycin and sodium bicarbonate to grazing lactating dairy cows. J Dairy Sci 82, 1545-1554.

Dirksen GU, MC Smith. 1987. Acquisition and analysis of bovine rumen fluid. Bovine Pract 22, 108-116.

Dohme F, TJ DeVries, KA Beauchemin. 2008. Repeated ruminal acidosis challenges in lactating dairy cows at high and low risk for developing acidosis: ruminal pH. J Dairy Sci 91, 3554-3567.

Duffield T, JC Plaizier, A Fairfield, R Bagg, G Vessie, P Dick, J Wilson, J Aramini, B McBride. 2004. Comparison of techniques for measurement of rumen pH in lactating dairy cows. J Dairy Sci 87, 59-66.

Garrett EF, MN Pereira, KV Nordlund, LE Armentano, WJ Goodger, GR Oetzel. 1999. Diagnostic methods for the detection of subacute ruminal acidosis in dairy cows. J Dairy Sci 82, 1170-1178.

Garry FB. 2002. Indigestion in ruminants. In: Smith BP (ed). Large Animal Internal Medicine. $3^{\text {th }}$. Mosby, St. Louis, USA, Pp 722-747.

Gianesella M, M Morgante, C Stelletta, L Ravorotto, E Giudice, R Van Saun. 2010. Evaluating the effects of ruminocentesis on health and performance in dairy cows. Acta Vet Brn 79, 459-468.

Kolver ES, MJ de Veth. 2002. Prediction of ruminal pH from pasturebased diets. J Dairy Sci 85, 1255-1266.

Maruta CA, MLdR Leal, D Mendes Netto, CS Mori, AC Antonelli, EL Ortolani. 2008. The measurement of urine $\mathrm{pH}$ to predict the amount of buffer used in the treatment of acute rumen lactic acidosis in cattle. Ciência Rural 38, 717-722.
Maruta CA, EL Ortolani. 2002. Suceptibilidade de bovinos das raças Jersey e Gir à acidose láctica ruminal: I-Variáveis ruminais e fecais. Ciência Rural 32, 55-59.

Morgante M, C Stelletta, P Berzaghi, M Gianesella, I Andrighetto. 2007. Subacute rumen acidosis in lactating cows: an investigation in intensive Italian dairy herds. J Anim Physiol Anim Nutr (Berl) 91, 226-234.

Murrell JC, EP Psatha, EM Scott, J Reid, LJ Hellebrekers. 2008. Application of a modified form of the Glasgow pain scale in a veterinary teaching centre in the Netherlands. Vet Rec 162, 403-408.

Nordlund KV, EF Garrett. 1994. Rumenocentesis: A technique for collecting rumen fluid for the diagnosis of subacute rumen acidosis in dairy herds. Bovine Pract 28, 109-112.

Noro M, J Borkert, GA Hinostroza, R Pulido, F Wittwer. 2011. Variaciones diarias de metabolitos sanguíneos y su relación con el comportamiento alimenticio en vacas lecheras a pastoreo primaveral. Rev Cient 21, 125-130.

Noro M, R Chihuailaf, J Céspedes, F Wittwer. 2010. Acidosis subaguda (SARA) y alcalosis ruminal en rebaños lecheros en pastoreo otoñal y primaveral. XXXV Congreso Sociedad Chilena de Producción Animal, Pp 193-194.

O'Grady L, ML Doherty, F Mulligan. 2008. Subacute ruminal acidosis (SARA) in grazing Irish dairy cows. Vet $J 176,44-49$.

Oetzel GR. 2003. Herd-based biological testing for metabolic disorders. Adv Dairy Technol 15, 275-285.

Scandolo D, M Noro, H Bohmwald, PA Contreras, F Wittwer. 2007. Variación diurna del $\mathrm{pH}$ y de las concentraciones de magnesio y potasio del fluido ruminal en vacas lecheras a pastoreo. Arch Med Vet 39, 141-146.

Wales WJ, ES Kolver, PL Thorne, AR Egan. 2004. Diurnal variation in ruminal $\mathrm{pH}$ on the digestibility of highly digestible perennial ryegrass during continuous culture fermentation. J Dairy Sci 87, 1864-1871.

Westwood CT, E Bramley, IJ Lean. 2003. Review of the relationship between nutrition and lameness in pasture-fed dairy cattle. $N Z$ Vet $J$ 51, 208-218.

Wittwer F. 2009. Análisis del líquido ruminal como ayuda diagnóstica en alteraciones en el rumen. In: Contreras PA, Noro M (eds). Rumen Morfofisiología, trastornos y modulación de la actividad fermentativa.

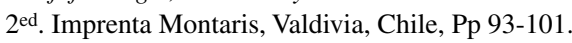

